\section{Deficiência de ferro: ainda a principal etiologia entre crianças encaminhadas por motivo de anemia para serviço especializado de hematologia}

\author{
Iron deficiency: still the main cause of \\ referral of children to hematology services \\ for reason of anemia
}

Márcio Antônio Portugal Santana 1

Rocksane de Carvalho Norton 2

Rachel Aparecida Ferreira Fernandes 3

\begin{abstract}
Objectives: to determine the reasons for diagnosis of anemia among children and referral to specialized services.

Methods: an historical and concurrent cohort study was carried out among anemic children referred to the Hematology Service of the Federal University of Minas Gerais's Clinical Hospital, Belo Horizonte, in the State of Minas Gerais, Brazil, between May 1999 and December 2001.

Results: 153 children aged under 16 were evaluated, $83 \%$ of whom had been referred by Health Centers. Approximately one third of the children did not receive any treatment at the referring service. One hundred and eleven (71.3\%) were diagnosed as having iron-deficiency anemia. Thirteen children (8.6\%) received a diagnosis of thalassemia minor; and a further thirteen other anemia-related diagnoses. $11.5 \%$ had no kind of anemia. The mean hemoglobin levels were significantly higher when tested by a hematologist than at the referring services (9.7 1.9 vs. 8.9 2.0 ); $p=0.001$. Only $16.2 \%$ of children did not respond to initial treatment with oral iron salts, with iron (II) sulfate being the medication usually prescribed $(79.3 \%)$.

Conclusions: iron deficiency was the main cause of children being referred to a specialized service for reason of anemia. Unnecessary referral of individuals with iron-deficiency anemia is potentially harmful to patients and puts an excessive burden on the health system.
\end{abstract}

Key words Anemia, iron-deficiency, Diagnostic errors, Treatment, Diagnosis
1-3 Departamento de Pediatria. Faculdade de Medicina. Universidade Federal de Minas Gerais. Av. Alfredo Balena, 190. $4^{\circ}$ andar. Belo Horizonte, MG, Brasil. CEP: 30.130-100.

E-mail: mapsbh@bol.com.br

\section{Resumo}

Objetivos: determinar os motivos de encaminhamento e diagnósticos de crianças com anemia para serviço especializado.

Métodos: coorte histórico e concorrente de crianças com anemia encaminhadas ao Serviço de Hematologia do Hospital das Clínicas da Universidade Federal de Minas Gerais, Belo Horizonte, Minas Gerais, Brasil, entre maio de 1999 e dezembro de 2001.

Resultados: foram avaliadas 153 crianças menores de 16 anos, sendo $83 \%$ procedentes de Centros de Saúde. Aproximadamente um terço das crianças não recebeu qualquer terapêutica no serviço de origem. Cento e onze (71,3\%) tiveram diagnóstico de anemia ferropriva. Treze crianças $(8,6 \%)$ tiveram diagnóstico de talassemia minor; outras treze crianças apresentaram outros diagnósticos de anemias e 11,5\% das crianças não apresentavam anemia. Os valores médios de hemoglobina na abordagem pelo hematologista foram significativamente maiores que os dos serviços de origem ( $9,7 \pm 1,9$ vs. $8,9 \pm 2,0) ; p=0,001$. Somente $16,2 \%$ crianças não responderam ao primeiro tratamento com sais de ferro oral, sendo o sulfato ferroso o principal medicamento prescrito $(79,3 \%)$.

Conclusões: a deficiência de ferro foi a principal etiologia das crianças encaminhadas por motivo de anemia para serviço especializado. Encaminhamentos desnecessários de indivíduos com anemia ferropriva para serviços especializados representam prejuizo aos pacientes e onus excedente para o sistema de saúde.

Palavras-chave Anemia ferropriva, Erros de diagnóstico, Tratamento, Diagnóstico 


\section{Introdução}

A anemia ferropriva é a anemia carencial mais frequente na prática clínica, pediátrica e hematológica, ${ }^{1}$ sendo mais prevalente em associação com baixos padrões socioeconômicos. ${ }^{2}$ Estudos relatam que crianças, adolescentes, gestantes e mulheres em idade fértil são os grupos de maior risco para desenvolverem anemia ferropriva, podendo a prevalência atingir $60 \%$ dos lactentes. 3,4 No Brasil, as taxas de prevalência de anemia variam de $16 \%$ a $35 \%$, sendo maiores nos Estados menos desenvolvidos e regiões não metropolitanas. 5

Embora a palidez seja o achado clínico mais importante, outras manifestações não hematológicas, como o atraso no desenvolvimento neuropsicomotor e a deficiência da resposta imunológica, têm sido verificados em crianças com ferropenia, mesmo antes do surgimento de anemia.6,7

A avaliação laboratorial é geralmente de fácil interpretação e obtenção, sendo o diagnóstico confirmado quando há presença de anemia microcítica e hipocrômica e exames de análise do ferro corporal sugestivos de ferropenia. 8 A história alimentar também é importante para se estimar o aporte de ferro da criança. 9

O tratamento é baseado na reposição de ferro por via oral ou parenteral, devendo a medicação oral ser administrada por quatro a seis meses para correção dos níveis de hemoglobina e reposição das reservas corporais de ferro. $8 \mathrm{O}$ tratamento é bem tolerado, com uma boa resposta ao final do primeiro mês, desde que administrado corretamente. 8

A facilidade do diagnóstico e a disponibilidade do tratamento nos centros de saúde permitem que a doença possa ser tratada, precoce e eficazmente, no âmbito da atenção primária de saúde. ${ }^{8,10}$

Este estudo teve como objetivos determinar a frequência de anemia ferropriva entre as crianças encaminhadas para investigação de anemia ao serviço secundário do Hospital das Clínicas da Universidade Federal de Minas Gerais (HC-UFMG) e identificar a origem e os fatores responsáveis pelos encaminhamentos ao serviço especializado, avaliando a resposta à terapêutica instituída.

\section{Métodos}

Trata-se de um estudo observacional, de base ambulatorial, realizado no Serviço de Hematologia Pediátrica do Hospital das Clínicas (HC) da Universidade Federal de Minas Gerais (UFMG), no qual os atendimentos são realizados por hematologistas pediátricos de crianças de 0 a 16 anos, do
Sistema Único de Saúde (SUS), agendados pela Prefeitura de Belo Horizonte, Minas Gerais. Foram estudadas 153 crianças com idade inferior ou igual a 16, anos cujas guias ou relatórios de encaminhamento continham a palavra "anemia" como justificativa para avaliação do hematologista, atendidas de maio de 1999 a dezembro de 2001.

Os dados foram coletados através de questionário específico: retrospectivamente a partir da análise dos prontuários médicos arquivados no Serviço de Arquivo Médico (SAME) do HC-UFMG e, prospectivamente, mediante entrevistas realizadas com os pais ou responsáveis durante as consultas habituais do Ambulatório de Hematologia Pediátrica. O Termo de Esclarecimento foi lido e as eventuais dúvidas discutidas antes da assinatura do Termo de Consentimento.

Foram excluídas crianças cujo motivo principal do encaminhamento não tenha sido anemia ou que apresentassem ferropenia isolada sem anemia.

Os dados laboratoriais dos pacientes nos serviços de saúde onde tinham sido anteriormente atendidos foram obtidos a partir dos exames trazidos pelo responsável da criança ou a partir das Guias de Referência de encaminhamento ao especialista.

Adotou-se como referência para esse estudo os valores da hemoglobina, do hematócrito, número de hemácias, volume corpuscular médio (VCM) e hemoglobina corpuscular média (HCM) escalonados por idade, visando aumentar a especificidade do diagnóstico de anemia devido às particularidade da faixa etária pediátrica (Tabela 1). $8,11 \mathrm{~A}$ anemia foi definida como a presença de concentração da hemoglobina abaixo dos valores mínimos de referência apresentados na Tabela 1 .

Foram determinadas as frequências de gênero, origem, motivo de encaminhamento, realização de exames complementares, dados clínicos e tratamentos realizados nos serviços de origem. Pacientes cujos encaminhamentos citavam outros motivos, além da anemia, foram classificados em outra categoria, denominada Anemia + outro motivo.

No serviço secundário de saúde do Hospital das Clínicas determinou-se a frequência dos diagnósticos, assim como dos tratamentos e resposta terapêutica.

Adotou-se como critério de resposta terapêutica a elevação igual ou superior a $1 \mathrm{~g} / \mathrm{dL}$ na concentração da hemoglobina após, pelo menos, um mês de tratamento no HC-UFMG, quando comparada com o nível de hemoglobina anterior ao uso de ferro medicamentoso. 12,13

Para fins de análise, a anemia foi categorizada nos seguintes grupos: 
Anemia ferropriva: concentrações de hemoglobina, volume corpuscular médio (VCM) e hemoglobina corpuscular média (HCM) abaixo do valor mínimo de referência para idade e sexo, somadas à presença de dois ou mais fatores de risco para desenvolvimento de ferropenia (prematuridade, gestação gemelar, peso ao nascimento inferior a $2500 \mathrm{~g}$, aleitamento materno inferior a quatro meses, ausência de suplementação profilática com ferruginosos, menarca ou fluxo menstrual aumentado) e/ou a presença de um ou mais exames de ferro corporal sugestivos de ferropenia (redução do ferro sérico, ferritina sérica, índice de saturação de transferrina e aumento da capacidade total de ligação de ferro).

Anemia em remissão: anemia no hemograma do serviço de origem, de crianças que receberam tratamento com ferro oral ou parenteral naquele serviço e elevação da hemoglobina acima dos valores de referência para idade e sexo no primeiro hemograma solicitado no Ambulatório de Hematologia Pediátrica. Essas crianças poderiam estar ou não em tratamento para anemia no dia da primeira consulta e apresentarem microcitose, hipocrômia ou ferropenia, porém com concentração de hemoglobina já normalizada.

$\beta$-Talassemia: elevação dos níveis de hemoglobina $\mathrm{A}_{2}$, acompanhado ou não do aumento da hemoglobina fetal na eletroforese de hemoglobina.

Ausência de anemia: hemoglobina acima do valor mínimo de referência para idade e sexo, de acordo com a Tabela 1 . As crianças que apresentavam ferropenia sem anemia poderiam estar nesse grupo, porém não foi objetivo desse estudo identificá-las.

Outros diagnósticos: as crianças anêmicas que não preencheram os critérios citados acima e/ou tiveram diagnóstico de outras anemias específicas.

As doses de sal ferroso utilizadas pela criança, de acordo com a prescrição feita pelo serviço de origem, foram calculadas em ferro elemento/Kg/dia, a partir das informações obtidas (informações do responsável ou de prescrições médicas) durante a primeira consulta no Ambulatório de Hematologia do HC-UFMG.

As diferenças entre as médias dos valores da hemoglobina, do hematócrito, número de hemácias, volume corpuscular médio (VCM) e hemoglobina corpuscular média (HCM) entre os serviços de origem e o HC-UFMG, antes e após tratamento, foram avaliados pelo teste de " $t$ " pareado.

A presença de associação entre o motivo de encaminhamento pelo serviço de origem e o diagnóstico firmado no HC-UFMG foi avaliada pelo teste do qui-quadrado exato, via Monte Carlo. O nível de significância estatística admitido foi de 5\%.

$\mathrm{O}$ projeto foi aprovado na Câmara do Departamento de Pediatria e pelo Comitê de Ética em Pesquisa da UFMG.

\section{Tabela 1}

Valores médios, mínimos e máximos para hemácias, hemoglobina, hematócrito, volume corpuscular médio e hemoglobina corpuscular média, de acordo com a idade e sexo para crianças e adolescentes.

\begin{tabular}{lccccc}
\hline Idade & Hemácias (milhões/mm3) & Hemoglobina (g/dL) & Hematócrito (\%) & VCM (fl) & HCM (pg) $^{*}$ \\
\cline { 2 - 6 } & $\bar{X}$ (min-max.) & $\bar{X}$ (min-max.) & $\bar{X}$ (min-max.) & $\bar{X}$ (min-max.) & $\bar{X}$ (min-max.) \\
\hline Recém-nascido & $4,7(3,9-5,5)$ & $16,5(13,5-19,5)$ & $51(42-60)$ & $108(98-118)$ & $34(31-37)$ \\
1 semana & $5,1(3,9-6,3)$ & $17,5(13,5-21,5)$ & $54(42-66)$ & $107(88-126)$ & $34(28-40)$ \\
1 mês & $4,2(3,0-5,4)$ & $14,0(10,0-18,0)$ & $43(31-55)$ & $104(85-123)$ & $34(28-40)$ \\
2 meses & $3,8(2,7-4,9)$ & $11,5(9,0-14,0)$ & $35(28-42)$ & $96(77-115)$ & $30(26-34)$ \\
3-6 meses & $3,8(3,1-4,5)$ & $11,5(9,5-13,5)$ & $35(29-41)$ & $91(74-108)$ & $30(25-35)$ \\
6 meses-2 anos & $4,5(3,7-5,3)$ & $12,0(10,5-13,5)$ & $36(33-39)$ & $78(70-86)$ & $27(23-31)$ \\
2-6 anos & $4,6(3,9-5,3)$ & $12,5(11,5-13,5)$ & $37(34-40)$ & $81(75-87)$ & $27(24-30)$ \\
6-12 anos & $4,6(4,0-5,2)$ & $13,5(11,5-15,5$ & $40(35-45)$ & $86(77-95)$ & $29(25-33)$ \\
$>12$ anos feminino & $4,6(4,1-5,1)$ & $14,0(12,016,0)$ & $41(36-46)$ & $90(78-102)$ & $30(25-35)$ \\
$>12$ anos masculino & $4,9(4,5-5,3)$ & $14,5(13,0-16,0)$ & $43(37-49)$ & $88(78-98)$ & $30(25-35)$
\end{tabular}

$\mathrm{VCM}=$ volume corpuscular médio; $\mathrm{HCM}=$ hemoglobina corpuscular média; *Fentolitro; † picograma.

Fonte: Adaptado de: Viana MB, Alvim RC. Anemias. In: Leão E. et al. Pediatria ambulatorial. Belo Horizonte; 1998.8 


\section{Resultados}

Das 153 crianças estudadas, em $131(85,7 \%)$ as informações foram obtidas por meio de entrevistas realizadas no momento da consulta, enquanto para 22 crianças $(14,4 \%)$ os dados foram obtidos retrospectivamente, a partir da análise dos prontuários.

A idade variou de 22 dias a 15 anos de vida, com média de 38 meses. Noventa e três crianças $(60,8 \%)$ eram do sexo masculino. A maioria, 127 crianças $(83 \%)$, foi encaminhada de ambulatórios e unidades básicas de saúde. Cerca de $74,5 \%$ era oriunda da Região Metropolitana de Belo Horizonte, sendo 97 crianças $(63,4 \%)$ do próprio município e $17(11,1 \%)$ dos demais municípios da região.

Os motivos dos encaminhamentos, conforme registrado nos prontuários ou nas guias de encaminhamento, estão representados na Tabela 2, sendo mais frequentes diagnóstico de anemia $(37,3 \%)$ e anemia sem resposta oral $(27,3 \%)$.

Trinta e três crianças $(21,9 \%)$ haviam realizado o exame hematológico que motivou o encaminhamento ao ambulatório de hematologia na rotina dos serviços, ou seja, sem uma indicação clínica precisa segundo anotação nas guias de referência ou informação do acompanhante. A palidez foi o principal sinal clínico associado à anemia, relatado em $28,7 \%$ dos casos.

Tabela 2

Motivos de encaminhamento do serviço de origem e diagnósticos das 153 crianças avaliadas por anemia. Hospital das Clínicas da Universidade Federal de Minas Gerais em Belo Horizonte.

\begin{tabular}{|c|c|c|c|c|c|c|c|c|c|c|c|c|}
\hline \multirow{3}{*}{$\begin{array}{l}\text { Serviço de Origem } \\
\text { Motivo de } \\
\text { Encaminhamento }\end{array}$} & \multicolumn{12}{|c|}{ Diagnósticos firmados no HC-UFMG* } \\
\hline & \multicolumn{2}{|c|}{$\begin{array}{l}\text { Anemia } \\
\text { ferropriva }\end{array}$} & \multicolumn{2}{|c|}{$\begin{array}{l}\text { Anemia ferropriva } \\
\text { em remissão }\end{array}$} & \multicolumn{2}{|c|}{$\begin{array}{l}\text { Ausência de } \\
\text { anemia }\end{array}$} & \multicolumn{2}{|c|}{ Talassemia minor } & \multicolumn{2}{|c|}{$\begin{array}{c}\text { Outros } \\
\text { diagnósticos }\end{array}$} & \multicolumn{2}{|c|}{ Total } \\
\hline & $\mathrm{n}$ & $\%$ & $\mathrm{n}$ & $\%$ & $\mathrm{n}$ & $\%$ & $\mathrm{n}$ & $\%$ & $\mathrm{n}$ & $\%$ & $\mathrm{n}$ & $\%$ \\
\hline Anemia & 37 & 24,1 & 5 & 3,3 & 9 & 5,9 & 3 & 2,0 & 3 & 2,0 & 57 & 37,3 \\
\hline Anemia grave & 9 & 5,9 & - & - & - & - & - & - & 2 & 1,3 & 11 & 7,2 \\
\hline Anemia e plaquetose & 3 & 2,0 & - & - & - & - & 1 & 0,6 & - & - & 4 & 2,6 \\
\hline Anemia microcítica/hipocrômica & 11 & 7,3 & 1 & 0,6 & 4 & 2,7 & - & - & - & - & 16 & 10,6 \\
\hline Anemia megaloblástica & - & - & - & - & - & - & - & - & 1 & 0,6 & 1 & 0,6 \\
\hline $\begin{array}{l}\text { Anemia sem resposta ao } \\
\text { ferro oral }\end{array}$ & 28 & 28,3 & 4 & 2,7 & 3 & 2,0 & 7 & 4,5 & - & - & 42 & 27,3 \\
\hline Anemia + outro motivo & 11 & 11,1 & - & - & 2 & 1,3 & 2 & 1,3 & 7 & 4,5 & 22 & 14,3 \\
\hline Total & 99 & 64,8 & 10 & 6,5 & 18 & 11,5 & 13 & 8,6 & 13 & 8,6 & 153 & 100,0 \\
\hline
\end{tabular}

* Hospital das Clínicas da Universidade Federal de Minas Gerais.

Os níveis médios de hemoglobina, volume corpuscular médio (VCM), hemoglobina corpuscular média (HCM) e índice de anisocitose (RDW) dos exames realizados nos serviços de origem e na primeira abordagem no HC-UFMG estão na Tabela 3, observando-se uma elevação significativa nos parâmetros laboratoriais com a evolução do tratamento das crianças.

Quarenta e quatro crianças $(28,7 \%)$ foram encaminhadas pelos serviços de origem sem qualquer tratamento para os baixos níveis de hemoglobina identificados.

Os valores de hemoglobina observados nos hemogramas realizados na primeira consulta no $\mathrm{HC}$ -
UFMG variaram de 3,8g/dL a $12,7 \mathrm{~g} / \mathrm{dL}$. Os diagnósticos das 153 crianças estão registrados na Tabela 2, verificando-se concordância entre os motivos de encaminhamentos feitos pelos serviços de origem e os diagnósticos firmados no HC-UFMG $(p=0,001)$.

Segundo os médicos que atenderam as crianças no serviço de atenção primária, 14,3\% apresentaram outras alterações nos exames laboratoriais e/ou clínicos, além da anemia, que motivaram a solicitação de avaliação por um especialista (Tabela 2). Ao revisar esses motivos foi observado que se tratava de sinais clínico-laboratoriais com possíveis associações a doenças mais complexas, tais como esferocitose, anemia hemolítica autoimune, anemia 
Valores médios de hemoglobina, volume corpuscular médio, hemoglobina corpuscular média e índice de anisocitose dos hemogramas realizados nos serviços de origem e à admissão no serviço de hematologia pediátrica do Hospital das Clínicas da Universidade Federal de Minas Gerais, em Belo Horizonte.

\begin{tabular}{lcccc}
\hline Parâmetro & \multicolumn{2}{c}{ Serviço de origem } & \multicolumn{2}{c}{$\begin{array}{c}\text { Hospital das Clínicas da Universidade } \\
\text { Federal de Minas Gerais }\end{array}$} \\
\cline { 2 - 5 } & $\mathrm{n}$ & $\overline{\mathrm{X}} \pm \mathrm{DP}$ & $\mathrm{n}$ & $\overline{\mathrm{X}} \pm \mathrm{DP}$ \\
\hline Hemoglobina $(\mathrm{g} / \mathrm{dL})$ & 139 & $8,9 \pm 2,0$ & 116 & $9,7 \pm 1,9$ \\
$\mathrm{VCM}^{*}$ & 115 & $68,2 \pm 12,8$ & 109 & $67,9 \pm 12,7$ \\
$\mathrm{HCM}^{* *}$ & 91 & $22,3 \pm 7,7$ & 82 & $22,4 \pm 7,2$ \\
$\mathrm{RDW}^{* * *}(\%)$ & 44 & $18,4 \pm 4,2$ & 63 & $19,0 \pm 4,4$ \\
\hline
\end{tabular}

* = volume corpuscular médio; ${ }^{*}=$ hemoglobina corpuscular média; *** = índice de anisocitose.

secundária à infecção, ovalocitose, deficiência de glicose 6-fosfato desidrogenase e leucemia linfoblástica, justificando, portanto, o encaminhamento para atendimento especializado.

Das crianças encaminhadas, $109 \quad(71,2 \%)$ tiveram o diagnóstico de anemia ferropriva confirmados no HC-UFMG. Entre estas, 10 (9,2\%) apresentavam níveis de hemoglobina dentro dos parâmetros de normalidade para idade e sexo. Duas apresentavam uma segunda causa de anemia além da ferropenia: esferocitose e anemia secundária a processo infeccioso agudo. Das 153 crianças encaminhadas ao serviço de hematologia do HC-UFMG, $111(72,5 \%)$ apresentavam anemia ferropriva com necessidade de terapêutica. No serviço de origem, 28
$(25,2 \%)$ crianças ferropênicas não receberam terapêutica antianêmica, $82(73,9 \%)$ foram tratadas e uma $(0,9 \%)$ recebeu hemotransfusão.

No HC-UFMG, o sulfato ferroso foi a principal formulação farmacêutica de ferro oral prescrito $(79,3 \%)$, sendo a dose de ferro elementar mais utilizada a de 3 a $4 \mathrm{mg} / \mathrm{kg} / \mathrm{dia}$ (83\%). Em 64 pacientes (58\%), a administração de sais de ferro foi prescrita adequadamente no horário antes das refeições.

A Tabela 4 mostra os valores médios de hemoglobina no serviço de origem em relação à primeira consulta no Hospital das Clínicas e esta em comparação à concentração de hemoglobina média ao final do estudo ou alta da criança.

Tabela 4

Análise da diferença dos valores de hemoglobina média das 153 crianças atendidas no Hospital das Clínicas da Universidade Federal de Minas Gerais.

\begin{tabular}{lccc}
\hline Hemoglobina $(\mathbf{g} / \mathbf{d L})$ & $\mathbf{N}$ & $\overline{\mathrm{X}} \pm \mathrm{DP}$ & Valor de $\boldsymbol{p}^{*}$ \\
\hline Hb origem & 139 & $8,9 \pm 2,0$ & - \\
Hb primeira consulta Hospital das Clínicas & 116 & $9,7 \pm 1,9$ & $<0,001$ \\
$\begin{array}{l}\text { da Universidade Federal de Minas Gerais } \\
\text { Hb final do tratamento no Hospital das } \\
\text { Clínicas da Universidade Federal de Minas Gerais }\end{array}$ & 139 & $11,4 \pm 1,1$ & $<0,001$ \\
\hline
\end{tabular}

* Teste " $\mathrm{t}$ " pareado comparaç̃̃es com $\mathrm{Hb}$ origem; $\mathrm{Hb}=$ hemoglobina. 
Das 111 crianças com anemia ferropriva, 93 $(83,8 \%)$ responderam ao primeiro tratamento. A mediana para o tempo de tratamento até a resposta para essas 93 crianças foi de 60 dias e o tempo total de tratamento teve mediana de 120 dias. Dezoito crianças $(16,2 \%)$ não responderam ao primeiro tratamento oral com ferro e necessitaram serem submetidas à nova investigação, ajuste da dose de ferro ou substituição da medicação, tendo a deficiência de ferro sido confirmada em todas elas. Erros na administração do ferro oral foram identificados em oito desses 18 pacientes (44,3\%). Em sete pacientes $(38,9 \%)$ foram identificados problemas associados como parasitoses e enterorragia e três (16,7\%) relato de intolerância à droga, tendo sido necessário sua substituição.

Apenas seis crianças $(5,4 \%)$ com diagnóstico confirmado de anemia ferropriva foram submetidas a tratamento com ferro parenteral e responderam satisfatoriamente ao tratamento, tendo apresentado elevação da concentração de hemoglobina superior a $1 \mathrm{~g} / \mathrm{dL}$.

\section{Discussão}

A anemia por deficiência de ferro é uma entidade classicamente diagnosticada e tratada pelo pediatra. 8,14 Viana et al.10 recomendam que a anemia ferropriva seja abordada em sua plenitude pelo médico da família, dispensando, inclusive, o acompanhamento do pediatra. Entretanto, $83 \%$ das crianças encaminhadas ao Serviço de Hematologia do HC foram oriundas das unidades básicas de saúde e $70,1 \%$ destas tiveram diagnóstico confirmado de anemia ferropriva. Stoltzfus e Dreyfuss 15 recomendam que pacientes com anemia ferropriva somente sejam encaminhados aos serviços de referência quando apresentarem níveis de hemoglobina menores que 7,0 g/dL. Alves e Fernandes 14 recomendam que o médico da família deve encaminhar crianças com anemia ferropriva ao hematologista nas seguintes situações: presença de fatores de risco para ferropenia juntamente com achados clínicos de outras doenças; falha no tratamento medicamentoso após afastar não adesão e fatores mantenedores da ferropenia; anemias hipocrômicas e microcíticas (ou não) com estudo de ferro corporal normal e baixos níveis de hemoglobina com instabilidade hemodinâmica e necessidade transfusional.

Houve concordância, na maioria dos casos, entre os diagnósticos do serviço de referência (HCUFMG) e os serviços de origem. Entretanto, em cerca de $10 \%$ dos pacientes foram observadas discordâncias entre o diagnóstico que motivou encaminhamento e o diagnóstico firmado no serviço ambulatorial de hematologia, tendo sido afastado o diagnóstico de anemia e, portanto, houve encaminhamento e atendimento desnecessários.

A ausência de resposta da anemia ao tratamento com ferro oral $(27,3 \%)$ foi o motivo principal do encaminhamento, porém, das crianças que tiveram diagnóstico confirmado de ferropenia na abordagem do $\mathrm{HC}$, a maioria $(78,1 \%)$ respondeu satisfatoriamente a um único tratamento oral realizado nesse serviço. Quando se analisou a terapêutica instituída, observou-se que a administração diária de sulfato ferroso oral foi o tratamento de eleição nos serviços de origem (70,3\%) e no HC-UFMG (79,3\%).

Apesar de não haver diferença significativa entre as doses utilizadas, apenas as crianças tratadas pelo especialista no HC-UFMG apresentaram resposta satisfatória ao primeiro tratamento instituído. Os motivos pelos quais essas crianças só obtiveram essa resposta no serviço especializado e os fatores responsáveis pela não resposta no serviço de origem não foram identificados. Fatores como insegurança em relação à definição diagnóstica e ao tratamento instituído, encaminhamento precoce antes de aguardar a resposta terapêutica no serviço de atenção primária ou mesmo a baixa adesão das crianças e responsáveis ao tratamento podem estar envolvidos na falta de resposta terapêutica nos serviços dos quais os pacientes eram provenientes. Essa última possibilidade foi bem levantada por Zlotkin et al. 16 como um dos fatores responsáveis por fracasso terapêutico em países desenvolvidos e em desenvolvimento.

Quando se analisou o tratamento instituído pelo serviço de origem para as crianças ferropênicas, mais de $70 \%$ havia sido encaminhada ao especialista após início de tratamento antianêmico. Novamente, a justificativa para o encaminhamento precoce destas crianças que já haviam iniciado o tratamento não foi identificado, apesar de a anemia ferropriva ser entidade nosológica comum da prática dos serviços básicos de saúde. $8,9,14$

Entre as crianças encaminhadas ao serviço de hematologia devido à má resposta ao tratamento com ferro oral, em aproximadamente $16 \%$ o diagnóstico de $\beta$-talassemia foi confirmado, justificando o encaminhamento. Porém, considerando que a talassemia constitui o principal diagnóstico diferencial da anemia ferropriva8,11 e outras anemias microcíticas e hipocrômicas, essa hipótese diagnóstica poderia ter sido levantada no serviço de origem, por meio da realização de eletroforese de hemoglobina, que evidencia a elevação dos níveis de hemoglobina $\mathrm{A}_{2} \cdot 11$ 
Considerando-se os valores médios dos parâmetros dos hemogramas encontrados nos serviços de origem, foi possível confirmar a presença de anemia hipocrômica e microcítica, com índice de anisocitose elevado (RDW). Sabe-se que o índice de anisocitose é um bom marcador para detectar a presença de anormalidades, mesmo quando analisado isoladamente, ${ }^{17}$ podendo ser usado tanto no diagnóstico diferencial da anemia ferropriva com talassemia, como para a avaliação de resposta ao tratamento com ferro. ${ }^{18}$

Quando se analisou o tratamento realizado nos serviço de origem, observou-se que os sais de ferro oral foram administrados para $68 \%$ das crianças, sendo o sulfato ferroso o mais prescrito. Um percentual de $41,1 \%$ das crianças recebeu doses de ferro elemento abaixo do recomendado 8 ( $3 \mathrm{mg}$ ferro elemento $/ \mathrm{kg} / \mathrm{dia})$. Somente $34,0 \%$ crianças receberam a medicação antes das refeições.

Chamou a atenção o fato de quase $30 \%$ das crianças com diagnóstico de anemia terem sido encaminhadas sem tratamento prévio e que em mais de $60 \%$ destas o diagnóstico de ferropenia foi confirmado, resultando, portanto, em atraso no início do tratamento específico da doença. Essas crianças, portanto, ficaram sujeitas a outras complicações não hematológicas.6,7 Das crianças que não foram submetidas a tratamento antianêmico nos serviços de origem, cerca de $20 \%$ não foram classificadas como anêmicas no HC-UFMG segundo os parâmetros utilizados nesse estudo, tendo sido, portanto, erroneamente diagnosticadas. $\mathrm{O}$ atendimento a pacientes da faixa etária pediátrica exige o conhecimento das variações fisiológicas dos parâmetros do hemograma, 8 tão particulares dessa fase da vida, a fim de evitar diagnósticos e tratamentos errôneos.
A concentração média da hemoglobina do primeiro hemograma realizado no $\mathrm{HC}$ foi significativamente maior do que os valores observados no serviço de origem. Esse dado evidencia que estas crianças já chegaram menos anêmicas ao especialista. Essa informação é facilmente explicada se observarmos que $71,3 \%$ das crianças já haviam recebido alguma forma de tratamento no serviço de origem, sendo a terapia com sais de ferro oral a mais frequente: $74 / 109$ crianças $(68,0 \%)$. Como $77 / 109$ crianças tratadas $(71,2 \%)$ realmente apresentavam ferropenia, elas responderam ao ferro oral e chegaram com maior nível de hemoglobina no HCUFMG. Vale ressaltar que $10 / 153$ crianças $(6,5 \%)$ já chegaram, inclusive, sem anemia (Tabela 2). É importante salientar que os exames dos serviços de origem foram realizados em laboratórios diferentes, podendo haver interferência de técnica e aparelhagem utilizada.

A concentração média de hemoglobina das crianças ao final do estudo foi de $11,4 \mathrm{~g} / \mathrm{dL}$, com elevação significativa quando comparada a hemoglobina do início do tratamento no HC-UFMG. Como a maioria das crianças eram ferropênicas e receberam tratamento ferruginoso com ótima resposta, observamos essa boa evolução do quadro.

Concluímos que a maioria das crianças encaminhadas por motivo de anemia para o serviço especializado de hematologia do HC-UFMG apresentava anemia ferropriva. Essa é uma patologia de fácil diagnóstico e com ótima resposta ao sulfato ferroso disponibilizado no SUS; esse fato poderia ter evitado o encaminhamento desnecessário, caso essas crianças tivessem sido eficazmente tratadas nos serviços de origem.

\section{Referências}

1. Olivares M, Walter T, Hertrampf E, Pizarro F. Anaemia and iron deficiency disease in children. Br Med Bull. 1999; 55: 534-43.

2. Neves MB, Silva EM, Morais MB. Prevalence and factors associated with iron deficiency in infants treated at a primary care center in Bélem, Pará, Brasil. Cad Saúde Pública. 2005; 21: 1911-8.

3. Pineda O, Ashmead, HD, Perez JM, Lemus CP. Effectiveness of iron amino acid chelate on the treatment of iron deficiency anemia in adolescents. J Appl Nutr. 1994; 46: 2-13.

4. Schneider JM, Fujii ML, Lamp CL, Lönnerdal B, Dewey $\mathrm{KG}$, Zidenberg-Cherr S. Anemia, iron deficiency and iron deficiency anemia in 12-36-mo-old children from lowincome families. Am J Clin Nutr. 2005; 82: 1269-75.
5. Norton RC, Figueredo RC, Diamante R, Leão E. Prevalence of anemia among school-children from Rio Acima (State of Minas Gerais, Brazil): use of the standardized prevalence method and evaluation of iron deficiency. Braz J Med Biol Res. 1996; 29: 1617-24.

6. Ghosh K. Non haematological effects of iron deficiency - a perspective. Ind J Med Sci. 2006; 60: 30-7.

7. Walter T, De Andraca I, Chadud P, Perales CG. Iron deficiency anemia: adverse effects on infant psychomotor development. Pediatrics. 1998; 84: 7-17.

8. Viana MB, Alvim RC. Anemias. In: Leão E, Corrêa JC, Viana MB, Mota JAC. Pediatria ambulatorial. Belo Horizonte: Coopmed; 1998. p. 589-99. 
9. Irwin JJ, Kirchner JT. Anemia in children. Am Fam Physician. 2001; 64: 1379-85.

10. Viana MR, Fernandes RAF. A criança com anemia ferropriva. In: Alves CRL, Alvim CG, Viana MRA. Atenção à saúde da criança. Belo Horizonte: Secretaria da Saúde de Minas Gerais; 2004. p. 131-5.

11. Lee GR. Iron deficiency and iron-deficiency anemia. In: Wintrobe MM, Lee GR. Wintrobe's clinical hematology. 10 ed. Baltimore: Willians \& Wilkins; 1999. v.1, p. 979-1010.

12. Wu AC, Lesperance L, Bernstein H. Screening for iron deficiency. Pediatr Rev. 2002; 23: 171-8.

13. Leung AK, Chan KW. Iron deficiency anemia. Adv Pediatr. 2001; 48: 385-408.

14. Alves CRL, Fernandes RAF. Anemia ferropriva. In: Alves CRL, Viana MRA. Saúde da família: cuidando de crianças e adolescentes. Belo Horizonte: Coopemed, 2003. p. 15964.

Recebido em 16 de maio de 2008

Versão final apresentada em 22 de junho de 2009

Aprovado em 30 de julho de 2009
15. Stoltzfus RJ, Dreyfuss ML. International Nutritional Anemia Consultative Group; World Health Organization; United Nations Children's Fund. Guildelines for the use of iron supplements to prevent and treat iron deficiency anemia. Washington: Int National Life Sci Press; 1998.

16. Zlotkin S, Antwi KY, Schauer C, Yeung G. Use of microencapsulated iron (II) fumarate sprinkles to prevent recurrence of anaemia in infants and young children at high risk. Bull World Health Organ. 2003; 81: 108-15.

17. Choi YS, Reid T. Anemia and red cell distribution width at the 12-month well-baby examination. South Med J. 1998; 91: 372-4.

18. Aslan D, Gümruk F, Gügey A, Altay, Ç. Importance of RDW value in differential diagnosis of hypochromea anemias. Am J Hematol. 2002; 69: 31-3. 